\title{
El rol de las creencias en contextos estratégicos: un estudio a través del género y las expectativas de los sujetos*
}

\author{
Ana León Mejía \\ Universidad Autónoma de Barcelona e IESA-CSIC \\ Luis M. Miller \\ Nuffield Centre for Experimental Social Sciences, \\ University of Oxford
}

\begin{abstract}
Resumen
El objetivo central de este trabajo es analizar el papel que desempeñan las creencias en contextos estratégicos. En concreto, estudiar de qué manera el género de los individuos interviene en el desarrollo de estas creencias. Es decir, si hombres y mujeres tienen distintas percepciones sobre la misma situación experimental, y sobre el comportamiento de otros sujetos. Para ello, analizamos comparativamente dos niveles: comportamiento y expectativas, viendo cómo interaccionan ambas dimensiones entre sí, y de qué manera el género de los sujetos es un mecanismo explicativo en dicha interacción.
\end{abstract}

Palabras clave: creencias de género, probabilidad subjetiva, riesgo.

Clasificación JEL: C72, C91, D64.

\begin{abstract}
This paper aims at analyzing the role played by beliefs in strategic situations. Specifically, we study how individuals' gender affects beliefs. That is, whether men and women perceive the same experimental situation and the behavior of other subjects differently. To do that, we compare two levels: behavior and expectations. We then analyze how these two dimensions interact with each other and how subjects' gender helps to explain such an interaction.

Keywords: gender beliefs, subjective probabilities, risk.

JEL classification: C72, C91, D64.

\section{Motivación}

El estudio de las creencias dentro de la economía experimental no ha recibido la misma atención que otros conceptos clásicos, como el de preferencia. Sin embargo, las creencias suponen un elemento central para comprender la motivación de los sujetos. En el caso de las diferencias de género en contextos experimentales, donde la literatura al respecto es amplia y las conclusiones arrojadas por las distintas investigaciones son dispares ${ }^{1}$ (Croson y Gneezy, 2009, en prensa), el análisis de las creencias se torna

* Este artículo se ha beneficiado de la concesión de un proyecto I+D financiado por el Ministerio de Educación y Ciencia y el FEDER, con referencia SEJ 2006-00959/SOCI.

${ }^{1}$ Distintos estudios experimentales encuentran diferencias significativas de género que apuntan a diferentes direcciones sin que, por ello, sea posible extraer un conocimiento firme de la magnitud y naturaleza de las mismas.
\end{abstract}


determinante. Nos permite dilucidar si hombres y mujeres tienen distintas expectativas acerca del comportamiento de otros sujetos y de qué manera perciben un contexto experimental. Es decir, podemos explorar qué sexo es más proclive a las variaciones experimentales (Cox y Deck, 2006; León y Miller, 2007) o cómo perciben el riesgo de sus propuestas o demandas. Por tanto, nos permite profundizar en el papel que desempeña el ambiente de un determinado juego o el carácter estratégico del mismo, y cómo ambos sexos perciben el riesgo que se deriva de la incertidumbre de este contexto.

Una gran cantidad de investigaciones se han centrado precisamente en el estudio de las diferencias entre hombres y mujeres en su aversión al riesgo, siendo uno de los pocos fenómenos sobre el que los distintos resultados apuntan a la misma dirección: la mayor aversión al riesgo del sexo femenino (Croson y Gneezy, 2009, en prensa; Eckel y Grossman, 1998, 2001; Byrnes y otros., 1999). Por desgracia no contamos con el mismo nivel de conocimientos sobre la función que desempeñan las creencias a la hora de evaluar el riesgo de un contexto estratégico. Tampoco contamos con evidencia suficiente como para entender de qué manera las expectativas o creencias, de naturaleza subjetiva, se corresponden efectivamente con la realidad objetiva. Es decir, de qué manera podrían percibir los sujetos un mayor o menor nivel de riesgo del que posee realmente una determinada interacción. Sin embargo, un estudio detallado de las creencias de los sujetos participantes en una investigación nos permite analizar comparativamente dos niveles: comportamiento y expectativas, viendo cómo interaccionan ambas dimensiones entre sí. Y por otra parte, podemos ver de qué manera el sexo de los sujetos es un mecanismo explicativo en dicha interacción. Es decir, si hombres y mujeres difieren tanto en el nivel de creencias como en el de conducta, y qué factores pueden intervenir o interaccionar con la variable de género.

Este trabajo persigue además contribuir a una vía abierta recientemente sobre el estudio de las diferencias de género en preferencias sociales, que considera tales diferencias como resultado, en parte, de la diferente sensibilidad de cada sexo al contexto experimental (Croson y Gneezy, 2009; Cox y Deck, 2006; Buchan y otros, 2008). En este caso, una exploración de las creencias de hombres y mujeres puede arrojar luz sobre la evaluación subjetiva que pudiera estar detrás de esta reacción diferencial. Esta línea de investigación es coherente con descubrimientos procedentes principalmente del ámbito de la neuropsicología y de la psicología cognitiva, que apuntan a diferencias cognitivas entre ambos sexos que intervienen en tareas muy específicas.

Teniendo en cuenta que la elaboración de creencias es una tarea cognitiva, vamos a estudiar la magnitud y naturaleza de las diferencias entre hombres y mujeres en dos contextos de distinto componente estratégico: el Juego del Ultimátum (Güth y otros, 1982) y el Juego del Yes-or-No (Gehrig y otros, 2007). Mediremos cómo influye en los participantes la información acerca del género del compañero con el que interactúa, y si el papel que juega esta información varía en función del sexo de los sujetos. Para ello usaremos un diseño factorial $(2 \times 2)$ formado por dos juegos y dos contextos estratégicos: Juego del Ultimátum con o sin información de género, y Juego del Yes-or-No con o sin tratamiento, y en los que interaccionan hombres y 
mujeres. Por último, cada participante ha de realizar una predicción sobre el comportamiento de su compañero o compañera siguiendo el método de obtención de creencias desarrollado por Schotter y Sopher (2007). Los resultados apuntan a que la información de género solo es relevante en ciertos contextos estratégicos y que los sujetos reflejan la percepción de este mayor nivel de riesgo en sus creencias.

La estructura de este trabajo es la siguiente. En el segundo apartado se describe el diseño experimental prestando especial atención al método usado para comunicar el género de los participantes sin introducir un efecto demanda en los mismos, y al procedimiento formal de obtención de creencias. En el tercer apartado presentamos los resultados relacionados con las creencias de los participantes, y su relación con la dimensión de comportamiento. El cuarto apartado resume los principales resultados y concluye este trabajo.

\section{Procedimiento y diseño experimental}

\subsection{Características generales del experimento}

Para tratar de responder a las preguntas de investigación planteadas anteriormente se llevó a cabo un experimento de laboratorio en el que participaron 376 estudiantes universitarios procedentes de diferentes estudios de licenciatura de la Universidad de Jena (Alemania). Estos fueron reclutados mediante ORSEE 2.0 (Greiner, 2004) y tomaron parte en un total de 12 sesiones experimentales en las que cada grupo estaba formado por el mismo número de hombres y mujeres ${ }^{2}$. Para la programación y conducción del experimento se utilizó el Software z-Tree (Fischbacher, 2007) en el laboratorio del Max Planck Institute of Economics.

Todos los sujetos que participaron en la investigación recibieron instrucciones por escrito que, además, fueron leídas en voz alta por un ayudante. Con ello se aseguró que todos los sujetos entendieran correctamente el contenido de las mismas. La comunicación entre sujetos no estaba permitida, resultando imposible identificar con qué otro estudiante se interaccionaba. El experimento se puso en marcha una vez que las instrucciones fueron leídas y las dudas de los sujetos resueltas en privado.

Para garantizar la distribución aleatoria de sujetos en los diferentes terminales del laboratorio, en la entrada del mismo se situaron dos cajas que contenían dos series de números: pares para los hombres e impares para las mujeres. Cada participante seleccionaba al azar un número de la caja correspondiente y se sentaba en el terminal que señalaba. Además de garantizar el anonimato de los participantes, así como su distribución aleatoria, este procedimiento permitió obtener la distribución deseada de parejas por género. Esta respondía a un diseño que combinaba género y roles: hombres interactuando con hombres $(\mathrm{HH})$, mujeres interactuando con mujeres

\footnotetext{
${ }^{2}$ Las instrucciones utilizadas en el experimento (originalmente en alemán) puede ser solicitadas a los autores.
} 
(MM), hombres interactuando con mujeres (HM) y mujeres interactuando con hombres $(\mathrm{MH})$. Al final de cada sesión experimental todos los sujetos recibieron el dinero en metálico que les correspondía de acuerdo a las ganancias obtenidas en cada juego, más un pago fijo por haber participado en el experimento de 2,5 euros.

Dos semanas antes del experimento, se realizó un estudio piloto en el que tomaron parte 32 sujetos y que tenía como objetivo detectar cualquier tipo de anomalía o deficiencia en el diseño experimental. Aunque la sesión fue llevada a cabo satisfactoriamente ésta sirvió para introducir cambios menores en el experimento realizado con posterioridad. Una de las diferencias entre ambas sesiones es que en el piloto los sujetos eran conscientes de que cada caja estaba destinada a uno de los dos géneros (contenían una marca distinta). Para evitar un efecto 'demanda' (que los sujetos se comportaran de manera acorde al tema de la investigación), en el experimento real se optó por retirar las etiquetas que marcaban cada urna. Análisis estadísticos posteriores demostraron que este cambio tuvo un efecto en la percepción de los participantes que pudimos constatar mediante el uso de un cuestionario post-experimental. Si en la sesión piloto 12 de cada 32 participantes, es decir, un 37,5\% de la muestra, adivinó que el tema del experimento era el género, en la sesiones correspondientes al experimento real tan solo 40 de los 376 (un 14\%) acertaron con la motivación del mismo. De ahí que los datos de la sesión experimental hayan sido excluidos del análisis final.

\subsection{Características sociodemográficas}

En cualquier experimento diseñado para estudiar el efecto del género en la interacción social y económica (gender pairing effect), uno de los aspectos más importantes es cómo comunicar el género de los participantes, sin que este hecho introduzca el efecto demanda que señalamos anteriormente. Por ello, en esta investigación se adoptó uno de los procedimientos descritos por Holm (2000), consistente en proporcionar la información de género contenida dentro de una descripción más amplia de los participantes. De este modo, la primera tarea del experimento consistía en contestar tres cuestiones que aparecían en la pantalla del ordenador:

- ¿Qué semestre estás cursando?

- ¿Eres de Jena?

- ¿Eres hombre o mujer?

La naturaleza de la primera pregunta era irrelevante y no tenía más finalidad que la de dificultar a los participantes adivinar la motivación del experimento. Por tanto esta última información no fue nunca suministrada a las distintas parejas. Por el contrario, la información sobre el origen del otro jugador fue proporcionada tanto en el grupo de control como en el grupo de tratamiento. Y por último, la información sobre el género, cuyo efecto deseábamos medir, solo fue suministrada en el grupo de tratamiento. 


\subsection{La información de género}

En las instrucciones se indicó a los participantes que debían de comprobar cuál era su rol en una tabla que se situaba en la esquina superior derecha de la pantalla del ordenador. La tabla estaba formada por dos columnas, denominadas «tu» $\mathrm{y}$ «tu compañero» y por dos filas: en una se indicaba el rol propio y el del compañero, y en la otra se proporcionaba una «información extra» sobre el origen y el género de ambos participantes (solo el origen en el grupo de control y el origen más el género en el de tratamiento). Por tanto, las cuatro posibles combinaciones que se obtenían en el grupo de tratamiento eran: «hombre de Jena», «hombre de fuera de Jena», «mujer de Jena», y «mujer de fuera de Jena». Mientras que en el grupo de control tan solo podía leerse «de Jena» o «de fuera de Jena».

Tal y como dijimos anteriormente, un aspecto que resultaba prioritario era conseguir que los sujetos no adivinaran cuál era el objetivo del experimento. Para constatar la consecución de este objetivo analizamos con detenimiento el cuestionario post-experimental. El resultado fue que tan solo un $14 \%$ de los participantes acertaron con la motivación identificando el género como la principal motivación del experimento, frente a una mayoría de sujetos que se decantaron por temas como el egoísmo, la justicia o la cooperación. Además, la proporción de participantes que eran conscientes del tema del experimento (el género) era la misma tanto en el grupo de control como en el de tratamiento (14,5\% y $12,5 \%$ respectivamente). Este dato es especialmente interesante a la hora de evitar el efecto confuso de los estereotipos de género sobre el tema del experimento, pues si, por ejemplo, una mayoría de sujetos hubiesen sido conscientes de la motivación experimental, esta investigación bien hubiera podido transformarse en una encuesta sobre el punto de vista sociopolítico de los sujetos, en lugar de una prueba sobre los cambios de comportamientos inducidos por la información de género.

\subsection{Estructura de los juegos}

En este experimento se empleó un diseño factorial $(2 \times 2)$ formado por dos juegos y dos contextos estratégicos. En cada una de las cuatro condiciones resultantes tomaron parte sujetos distintos (between-subjects design). El primero de ellos era la versión estándar del Juego del Ultimátum (Güth y otros, 1982) con información de género (UGT) en la que participaron un total de 128 sujetos. El segundo era una sesión de control del Ultimátum sin información de género (UGC), con un total de 64 sujetos. Y el tercer y cuarto escenario consistieron en el Juego del Yes-or-No (Gehrig y otros, 2007), con y sin información de género (YNT y YNC) en los que participaron un total de 120 y 64 estudiantes respectivamente. La Tabla 1 describe las principales características del experimento. 
TABLA 1

DISEÑO EXPERIMENTAL

\begin{tabular}{|c|c|c|c|c|}
\hline & UGT & UGC & YNT & YNC \\
\hline N. $^{\circ}$ de sesiones & 4 & 2 & 4 & 64 \\
\hline N. $^{\text {o de participantes }}$ & 128 & 64 & 120 & YNG \\
\hline Fecha & \multicolumn{3}{|c|}{ Junio-julio 2007 y enero 2008} \\
\hline Juego & UG & UG & YNG & No \\
\hline Tratamiento & Sí & No & Sí & $8,62 €$ \\
\hline Ganancia media & $8,29 €$ & $8,27 €$ & $8,82 €$ & . \\
\hline
\end{tabular}

En ambos juegos el jugador 1 decide cómo han de repartirse 100 ECUs (10 ECUs = 1 euro) entre él o ella y el jugador 2 . El jugador 1 puede elegir entre nueve distribuciones diferentes ([10-90], [20-80], [30-70], [40-60], [50-50], [60-40], [70-30], [80-20], [90-10]). La realización de este reparto, sin embargo, está condicionada a la decisión tomada por el jugador 2, de modo que si este último acepta el reparto, éste se lleva a cabo tal y como ha estipulado el jugador 1, y en el caso contrario, ambos jugadores se quedan sin ganancias. La decisión de los jugadores 2 sigue el método estratégico diseñado por Selten (1967) que consiste en tener que aceptar o rechazar cada una de las posibles ofertas potenciales, de manera que es posible obtener la oferta mínima que un determinado sujeto estaría dispuesto aceptar (minimum acceptable offer o MAO). A diferencia del UG, en el YNG los jugadores 2 tienen que decidir si aceptan o rechazan la oferta antes de conocer ésta.

\subsection{Obtención de creencias}

Después de jugar una sola ronda de uno de los dos juegos, a cada participante se le pidió que predijera el comportamiento de su compañero o compañera. El procedimiento para obtener las creencias que se siguió en ambos juegos es el desarrollado por Schotter y Sopher (2007), que consiste en preguntar, en el caso de los jugadores 2, por la probabilidad que asignan a recibir cada uno de los repartos potenciales. Y para los jugadores 1, la probabilidad de que su oferta sea aceptada por los jugadores $2^{3}$. A continuación se describen brevemente los procedimientos de obtención de creencias para los jugadores 1 y 2 .

\footnotetext{
${ }^{3}$ Este es exactamente el método seguido en el UG. En el caso del YNG el procedimiento es técnicamente el mismo pero la probabilidad que se pregunta a los sujetos no se refiere a la aceptación de una oferta en concreto, sino a a que los jugadores 2 digan sí.
} 


\subsubsection{PROCEDIMIENTO DE OBTENCIÓN DE CREENCIAS PARA LOS JUGADORES 2}

Sea $r=\left(r_{1}, r_{2}, r_{3}, r_{4}, r_{5}, r_{6}, r_{7}, r_{8}, r_{9}\right)$ el vector de creencias señaladas por cada jugador 2. Estas son las expectativas que tiene el jugador 2 de recibir cada una de las ofertas potenciales $(90,80,70,60,50,40,30,20,10)$. Dado que sólo una de estas cantidades será realmente propuesta, el pago del jugador 2 cuando la cantidad es seleccionada será:

$$
\Pi_{m}=20.000-\left\{\left(100-r_{m}\right)^{2}+\sum_{k \neq m}\left(r_{k}\right)^{2}\right\}
$$

Esta función debe entenderse del siguiente modo. Un jugador 2 comienza con 20.000 puntos y revela un vector de creencias $r=\left(r_{1}, r_{2}, r_{3}, r_{4}, r_{5}, r_{6}, r_{7}, r_{8}, r_{9}\right) . \mathrm{Si}$ su compañero o compañera (jugador 1 ) decide enviar la cantidad $m$, el jugador 2 ganaría el máximo de puntos si hubiera asignado toda la probabilidad a $m$. El hecho de que solo le asigne la probabilidad $r_{m}$ significa que ha cometido un error. Para penalizar este error, substraemos la cantidad de los puntos inciales. Además, el sujeto también es penalizado por la cantidad asignada a las otras ocho ofertas potenciales. Para ello, también substraemos $\left(r_{k}\right)^{2}$ de sus puntos. El peor de los casos, es decir cuando uno asigna toda la probabilidad a una oferta y el jugador 1 selecciona otra, supone una pago de $0 €$ en esta fase del experimento. En definitiva, decir la verdad es una estrategia óptima ${ }^{4}$.

\subsubsection{PROCEDIMIENTO DE OBTENCIÓN DE CREENCIAS PARA LOS JUGADORES 1}

El jugador 1 sólo introduce una probabilidad $\left(\Pi_{a}^{k}\right)$, donde $k$ representa el índice de una de las nueve ofertas potenciales. Esta es la probabilidad de que la oferta propuesta sea aceptada. Además, definimos $\left(\Pi_{r}^{k}\right)$ como la probabilidad complementaria, que la oferta sea rechazada. Como en el caso de los jugadores 2, el pago es calculado mediante una regla de pagos cuadrática. Así, el pago de los jugadores 1 en esta fase del experimento viene determinado por la siguiente fórmula:

$$
\Pi_{k}=20.000\left\{\left(100-\Pi_{a}^{k}\right)^{2}+\left(\Pi_{r}^{k}\right)^{2}\right\}
$$

En otras palabras, si la oferta es aceptada pero el jugador 1 predice que esta sólo sería aceptada con probabilidad $\Pi_{a}^{k}$, la función de pagos lo penaliza substrayendo $\left(100-\Pi_{a}^{k}\right)^{2}$ de sus puntos iniciales. También substrae $\left(\Pi_{r}^{k}\right)^{2}$ dado que esta es la pro-

\footnotetext{
${ }^{4}$ En este estudio utilizamos exactamente la misma regla de puntuación (scoring rule) usada por SCHOTTER y SOPHER (2007). Para calcular los pagos de nuestros participantes en ECUs, dividimos la cantidad resultante de la regla por 1.000. En las instrucciones del experimento la regla se explicaba de forma intuitiva y se ofrecía la posibilidad de ver la fórmula a quien la solicitara. Sólo 8 de los 376 participantes pidió ver la función. De este modo, le garantizamos a los participantes que cuanto mejor fuera su predicción mayor sería su pago sin necesidad de que entendieran en componente matemático de la fórmula.
} 
babilidad de que la oferta sea rechazada, lo cual no es el caso. Un pago similar puede ser definido si la oferta fuera rechazada.

Ambos tipos de jugadores fueron retribuidos usando las reglas de puntuación descritas anteriormente, las cuales garantizan que cuanto más cercanas son sus predicciones a la acción tomada por el otro jugador mayor es el pago recibido. El mecanismo empleado en este experimento constituye una buena herramienta para obtener las creencias verdaderas de los participantes en la investigación. Además, se aseguró que la cantidad de dinero que podía ser potencialmente recibida no fuese demasiado grande como para introducir cambios en las pautas de comportamiento de los participantes. Así, mientras que los participantes podían ganar un máximo de 100 ECUs en la fase de toma de decisiones, solo podían recibir 20 ECUs por la tarea predictiva.

\section{Resultados}

\subsection{El juego del Ultimátum}

En este apartado analizamos el papel que desempeñan las creencias individuales en contextos estratégicos. En concreto, nos centramos en el modo en el que el sexo de los individuos interviene en el desarrollo de estas creencias. Para ello debemos distinguir entre:

- Diferencias relacionadas con el sexo propio: cómo hombres y mujeres pueden albergar percepciones subjetivas distintas frente a una misma situación o contexto.

- Diferencias que emergen al conocer el sexo del compañero (gender pairing effect): cómo esta información de género puede influir en la formación de creencias sobre el riesgo que implica la decisión económica a la que se enfrentan.

Si la información de género influye a la hora de predecir el comportamiento de un sujeto, este hecho podría deberse a la influencia de estereotipos tales como que las mujeres son más generosas o los hombres implacables, etc., que pueden afectar a los sujetos que manejen tales configuraciones mentales. Antes de centrarnos en el estudio de las creencias, presentaremos algunos datos de comportamiento de los individuos que tomaron parte en el experimento.

Los resultados de comportamiento obtenidos están en línea con otros experimentos que analizan el UG. La oferta media, agrupando todas las sesiones del UG $(N=96)$, fue de 42,7 ECUs, la moda 50 ECUs y la mediana 40 ECUs. Básicamente podemos distinguir dos grupos distintos de jugadores 1: aquellos que ofrecen exactamente el $50 \%$ de la asignación inicial, y aquellos que intentan aprovecharse de ser los primeros en decidir (first-mover advantage) y ofrecer menos de la mitad ${ }^{5}$. En el caso de los jugadores 2, la tasa de rechazo, una vez cruzadas las ofertas de los jugadores 1 con el umbral de aceptación de los jugadores 2, es de un 8,3\%.

\footnotetext{
${ }^{5}$ Ver BRAÑAS y MILLER (2008) para una justificación mas amplia de esta division entre tipos de jugadores.
} 


\subsection{Creencias de género en los proposer}

Para analizar el comportamiento de los jugadores 1 compararemos dos grupos de individuos: aquellos que ofrecen la mitad de la asignación inicial $(n=41)$ y aquellos que ofrecen menos de la mitad $(n=50)$. Además, se ha descartado del análisis aquellos que ofrecieron más de la mitad $(n=5)$.

En concreto examinaremos la probabilidad subjetiva que los individuos asignan a que su oferta sea aceptada en ambos grupos (PSA). Esta es una medida de las expectativas que tienen los sujetos sobre el éxito del reparto propuesto, y que por otra parte, nos proporciona información sobre la percepción (subjetiva) del riesgo de rechazo de la oferta ${ }^{6}$. Debemos reiterar que se trata de una cuestión subjetiva que puede corresponderse o no con la realidad, y que es, al fin y al cabo, el escenario que los sujetos dibujan en sus mentes.

En la figura 1 podemos ver cuál es la probabilidad subjetiva de aceptación (PSA) de aquellos individuos que ofrecieron la mitad en contraposición a los que ofrecieron menos de la mitad.

\section{FIGURA 1 \\ MEDIANA DE LAS PSA POR GÉNERO Y TRATAMIENTO}

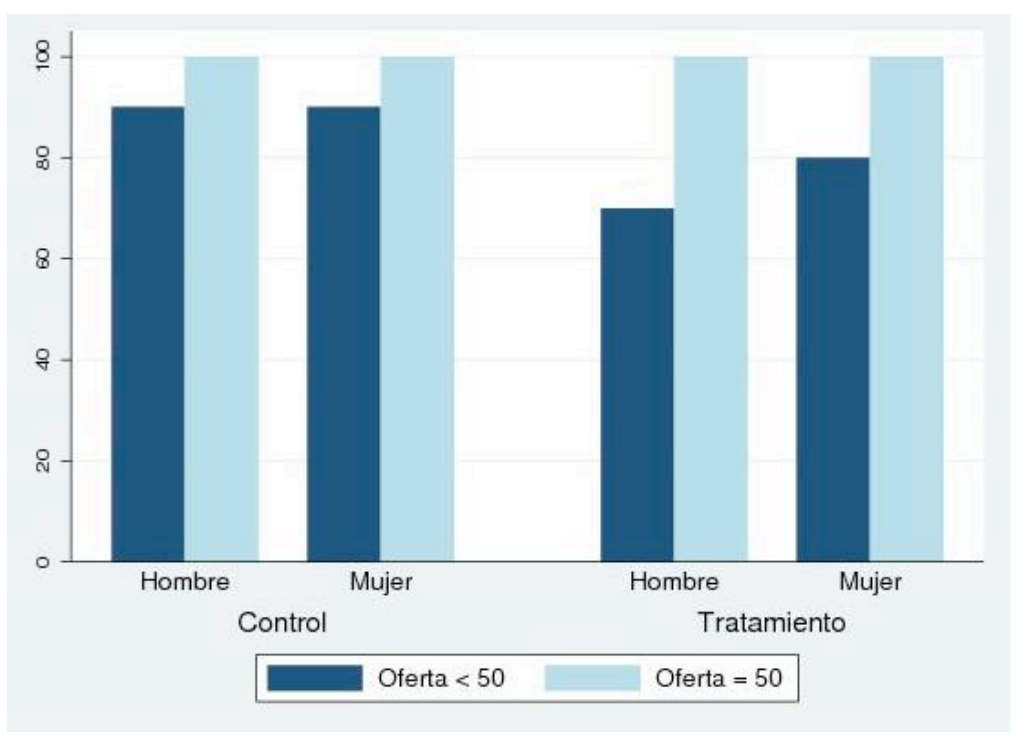

Como se puede observar, tanto hombres como mujeres que proponen una oferta igualitaria, y con independencia del tratamiento - de tener o no la información de género- están completamente seguros de que su oferta será aceptada (PSA $=100 \%)$.

\footnotetext{
${ }^{6}$ Es decir, resulta lógico pensar que, a medida que los sujetos atribuyen o perciben más riesgo de rechazo para una determinada oferta, la probabilidad que asignan a que ésta sea aceptada es menor.
} 
Sin embargo, cuando los jugadores 1 deciden sacar ventaja de su rol y proponer una oferta inferior, la PSA decrece. Y en este último caso sí que encontramos diferencias por género y por grupos de control y tratamiento que conviene analizar.

La PSA de ofertas desigualitarias es menor en el grupo de tratamiento que en el grupo de control: tanto hombres como mujeres cuando conocen el género del jugador con el que interaccionan asignan una PSA menor que cuando no lo conocen. Y se trata de una diferencia que es estadísticamente significativa (test de Mann-Whitney; $z=1,977 ; p=0,048)$. Sin embargo, los hombres del grupo de tratamiento atribuyen una probabilidad ligeramente menor a que la oferta sea aceptada que la que asignan las mujeres de su mismo grupo aunque en este caso la diferencia no es significativa.

Hasta el momento hemos analizado tan solo las expectativas o creencias de naturaleza subjetiva que albergan los sujetos, pero ¿coinciden estas con la realidad? La forma más sencilla de responder esta pregunta es comparar tales creencias con lo acontecido en el juego. Para ello introducimos una nueva medida que refleja el nivel objetivo de aceptación (NOA) de una determinada oferta. Se trata de analizar el comportamiento que han seguido los jugadores 2 a la hora de aceptar propuestas iguales a la mitad o propuestas inferiores a esta. Es decir, de contabilizar cuántos sujetos han aceptado propuestas igualitarias y cuántos propuestas inferiores ${ }^{7}$.

Por tanto se trata de contraponer la respuesta que anticipan los jugadores que no tienen información sobre lo que han hecho sus compañeros, con lo que efectivamente han decidido estos últimos. En la siguiente figura podemos ver de manera gráfica esta comparación entre el nivel subjetivo de las creencias de los jugadores 1 , y la objetividad que nos proporciona el análisis del comportamiento seguido por los jugadores 2.

\section{FIGURA 2 \\ MEDIANA DE LAS PSA Y NOA POR TRATAMIENTO Y TIPO DE PROPUESTA}

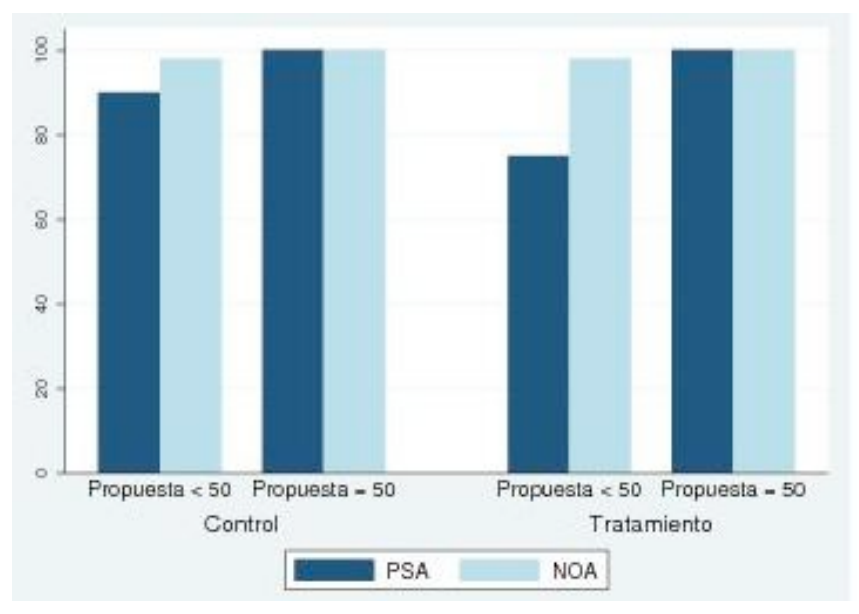

\footnotetext{
${ }^{7}$ De este modo mientras una oferta de 50 ECUs es aceptada por todos los sujetos, una oferta de 10 ECUs es aceptada solo por 45 de los 96 individuos, es decir, por un $47 \%$ de los mismos, y por tanto este es el nivel de aceptación o NOA de esta determinada oferta.
} 
Cuando analizamos las ofertas iguales a 50 ECUs, nos encontramos con que las expectativas subjetivas sobre aceptación y la realidad objetiva coinciden entre sí. Es decir, los jugadores 1 anticipan de manera acertada que su oferta no será rechazada por los jugadores 2 .

Las diferencias emergen de nuevo en el caso de las ofertas menores a la mitad. Aquí, apreciaciones subjetivas y comportamiento objetivo no coinciden, puesto que los jugadores 1 erran al predecir que sus compañeros 2 van a rechazar su oferta en un grado mayor al que realmente lo hacen. Dicho de otro modo, la NOA de ofertas menores a 50 desciende ligeramente con respecto al caso de la oferta igualitaria, mientras que la PSA asignada a ofertas desigualitarias desciende en gran medida con respecto a la PSA de las ofertas igualitarias. Además, esta diferencia es más acusada en el grupo de tratamiento, al que se le ha proporcionado la información de género, que en el grupo de control. Es decir, si el nivel de aceptación de ofertas desigualitarias es prácticamente igual en ambos grupos, lo mismo no puede afirmarse de las expectativas de ambos: los individuos del grupo de tratamiento atribuyen una probabilidad más alta de rechazo a su oferta que los individuos del grupo de control, siendo esta diferencia estadísticamente significativa (test de Mann-Whitney; $z=2,277 ; p=0,022$ ).

Por tanto, el escenario al que se van a enfrentar los sujetos (sobre la aceptación de sus ofertas) es idéntico en ambos casos, sin embargo los individuos imaginan escenarios diferentes dependiendo de si conocen o no el género de su compañero. Esto nos desvela que la información de género que se administra en el grupo de tratamiento influye en el nivel de creencias de los jugadores 1 aunque no en el de comportamiento de los jugadores 2. Más adelante profundizaremos sobre este aspecto, pero antes de ello debemos analizar si esta confrontación entre percepción (subjetiva) y realidad (objetiva) opera de igual manera en hombres y mujeres.

\section{FIGURA 3 \\ MEDIANA DE LAS PSA Y NOA POR GÉNERO Y TIPO DE PROPUESTA}

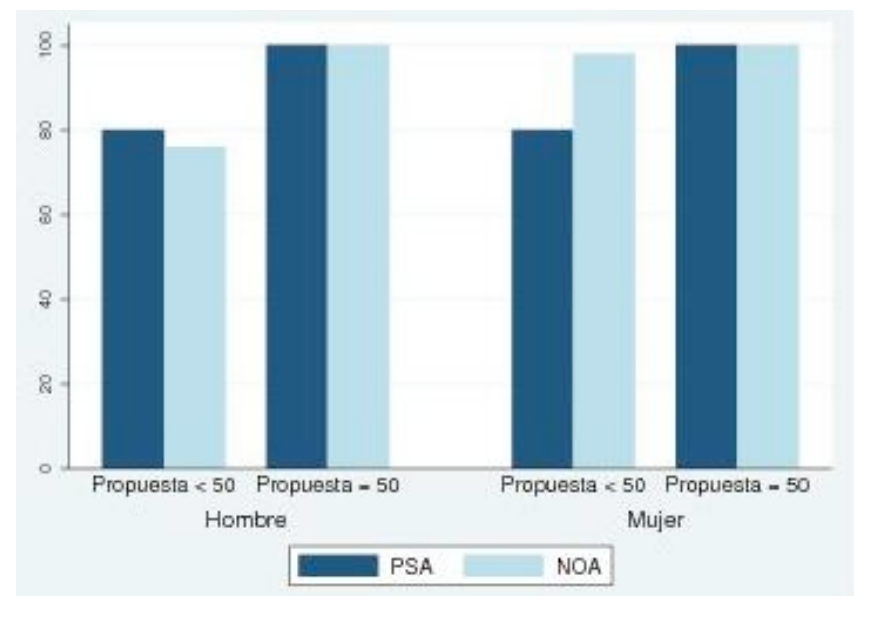


Al observar la figura 3 vemos como emergen diferencias al respecto, sin embargo, tales diferencias no surgen en el nivel subjetivo, sino que hombres y mujeres se enfrentan a niveles objetivos de aceptación distintos (test de Mann-Whitney; $z=-2,151 ; p=0,031)$. La diferencia entre ambos sexos no reside esta vez en las probabilidades que asignan los jugadores 1 a sus ofertas (cuyo comportamiento analizamos en este apartado), puesto que son similares, sino que residen en el comportamiento que siguen los jugadores 2, que aceptan con mayor medida las ofertas que han realizado las mujeres -dado que estas han sido mayores-. Este hecho se produce con independencia de que los jugadores 2 conozcan o no el género de su compañero. Es decir, a la hora de conformar cuál es la realidad objetiva con la que se enfrenta cada sexo, hemos agrupado a los jugadores 2 que han interaccionado solo con hombres y mujeres respectivamente, y con independencia de si pertenecían al grupo de control y tratamiento (de si conocían el género de su compañero), y tras ello hemos analizado cuál fue la respuesta de los mismos a las ofertas propuestas por cada sexo (se calcula de nuevo los niveles de aceptación de las ofertas iguales e inferiores a 50 ECUs pero discriminando esta vez por sexo del jugador 1).

Esta agrupación de jugadores 2 por sexo del jugador 1 es totalmente aleatoria al haber sido realizada con posterioridad al experimento, luego hombres y mujeres de ambos grupos se distribuyen por igual en ambos grupos. De manera que, esta medida del nivel objetivo de aceptación con el que se va a enfrentar los jugadores 1 de cada sexo, no se ve influenciada por ningún factor que, a priori, pueda determinar el comportamiento de los jugadores 2 en ambos grupos de interacción.

\subsection{Creencias de los jugadores 2 en el $U G$}

En primer lugar, hemos analizado si los jugadores 2 anticipan correctamente las propuestas realizadas por los jugadores 1 (NOR). Y el resultado obtenido es que los jugadores 2 sí realizan un cálculo acertado del tipo de oferta que van a recibir (PSR). Y este resultado es independiente del tratamiento y del sexo del participante (ver figura 4).

\section{FIGURA 4 \\ MEDIANA DE LAS PSR Y NOR POR GÉNERO Y TRATAMIENTO}

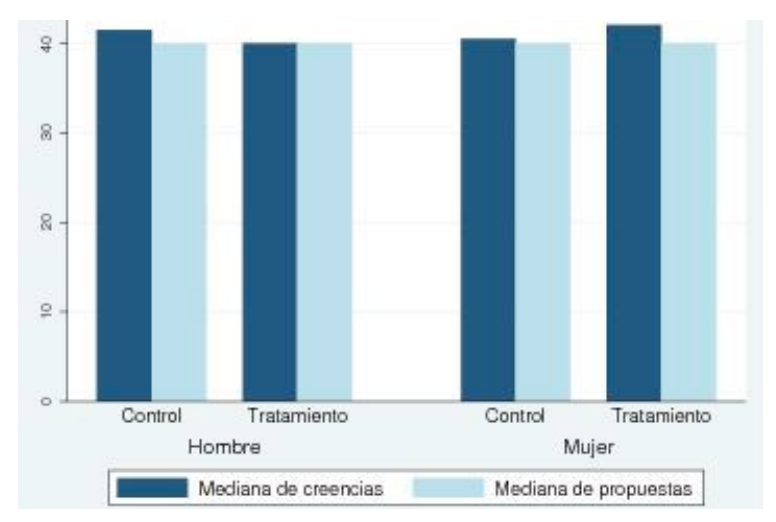


En el caso de los jugadores 2 en el UG, la única diferencia significativa en el plano de las creencias que encontramos es entre aquellos que están dispuestos a aceptar cualquier oferta $(n=45)$ y aquellos que demandan al menos el $20 \%$ del reparto $(n=51)^{8}$. Aquellos cuya $M A O>10$ creen que van a recibir una oferta mayor que aquellos cuya $M A O=10$, y esta diferencia es estadísticamente significativa (test de Mann-Whitney; $z=2,064 ; p=0,0390$ ). Asumiendo que creencia y decisiones son independientes, podemos afirmar que los jugadores 2 en el UG toman decisiones racionales de acuerdo con sus creencias, y que estas decisiones no están afectadas por la información extra que se les proporciona, en este caso el sexo de la persona con la que están interactuando.

\subsection{Síntesis de resultados para el $U G$}

- Los jugadores 1 que proponen ofertas igualitarias (50 ECUs) anticipan correctamente la respuesta de los jugadores 2 , con independencia de la información de género o de su sexo.

- Los jugadores 1 del grupo de tratamiento que proponen ofertas desigualitarias (menores a 50 ECUs), y con independencia de su sexo, se ven afectados por la información de género pues asignan una probabilidad de aceptación menor.

- Los jugadores 1, con independencia de su sexo, se enfrentan a niveles de aceptación distintos aunque la probabilidad subjetiva que asignan es idéntica.

- Los jugadores 2, con independencia del sexo y el tratamiento, asignan probabilidades subjetivas similares de recibir cada reparto potencial.

- Los jugadores 2 que aceptan cualquier oferta creen que van a recibir una cantidad significativemente inferior a aquellos que demandan más de la oferta mínima.

\subsection{El juego del Yes-or-No}

El juego del Yes-or-No es una versión extrema del juego del Ultimatum en la que los jugadores 2 tienen un poder de veto no condicionado (Gehrig y otros, 2007), es decir, solo pueden aceptar o rechazar una oferta sin conocer la naturaleza de la misma, o sin considerar la cuantía de cada uno de los repartos posibles que puedan ofrecer los jugadores 1 . Por ello, este juego es de una gran utilidad a la hora de evaluar el rol del componente estratégico del UG que desaparece en esta nueva estructura, mediante una comparación de lo que sucede en ambos escenarios. De igual modo, es posible realizar una comparación entre el YNG y el Juego del Dictador (DG). El YNG es una extensión más natural del UG que el DG ya que los jugadores 2 desempeñan un papel en el juego, aunque este sea muy limitado (aceptar o no con antelación la propuesta). Los estudios que han utilizado con anterioridad el

\footnotetext{
${ }^{8}$ De nuevo, una justificación del porqué de esta división puede ser encontrada en BRAÑAS y MILLER (2008).
} 
YNG han encontrado dos regularidades que merece la pena comentar. Primero, si comparamos los tres juegos entre sí, las ofertas realizadas en el YNG son menores que las del UG y mayores que las del DG. Segundo, los jugadores 2 en el YNG siempre aceptan la oferta (Gehrig y otros, 2007; Chlass y otros, 2008).

En este experimento encontramos el mismo resultado que los anteriores investigadores: todos los jugadores 2 aceptan el reparto y las ofertas que se realizan son significativamente inferiores a las del UG. A la hora de adentrarnos en el estudio comparativo de las creencias de los sujetos participantes en ambos juegos vemos como emergen algunas diferencias. Como argumentaremos más adelante, éstas son debidas principalmente a la eliminación del factor estratégico en el YNG que acabamos de mencionar.

\subsection{Creencias de los jugadores 1 en el $Y N G$}

La oferta media propuesta en el YNG es considerablemente menor que en el UG: 25,4 ECUs, con una mediana de 20 ECUs y una moda de 10 ECUs $(n=96)$. A la hora de analizar la PSA de los jugadores 1 y cómo esta medida difiere en un contexto no estratégico, lo primero que encontramos es que los jugadores 1 son conscientes de la falta de veto de los jugadores 2 y por tanto, además de realizar repartos inferiores (en el nivel de comportamiento), atribuyen una probabilidad muy alta a que su oferta sea aceptada. La mediana de la probabilidad de aceptación de la oferta es de 0,98 en el YNG. Es decir, anticipan acertadamente el comportamiento de los jugadores 2.

A continuación, analizaremos qué factores podrían tener una influencia en las creencias de los participantes. Para ello, de nuevo compararemos aquellos sujetos que ofrecen 10 ECUs $(n=45)$ con aquellos que ofrecen más de esta cantidad $(n=51)$. En este juego ofrecer mas de 10 ECUs solo puede ser explicado por algún tipo de función de utilidad que incorpore preferencias sociales, ya que el componente estratégico o de riesgo no desempeña ningún papel.

La mediana de la distribución de probabilidades subjetivas de aceptación de aquellos que ofrecen mas de 10 ECUs es de 0,9, y la de aquellos que ofrecen 10 ECUs es 1. Además, no existen diferencias significativas entre ambos grupos. Esto muestra como la creencia acerca de la probabilidad de aceptación de la oferta es independiente de la oferta misma en el YNG.

A la hora de explorar el papel desempeñado por el género de los participantes o por la información de género suministrada en el grupo de tratamiento en la asignación de PSA nos encontramos con que no existen diferencias atendiendo a estas dos variables (ver figura 5). 


\section{FIGURA 5 \\ MEDIANA DE LAS PSA Y NOR POR GÉNERO Y TRATAMIENTO}

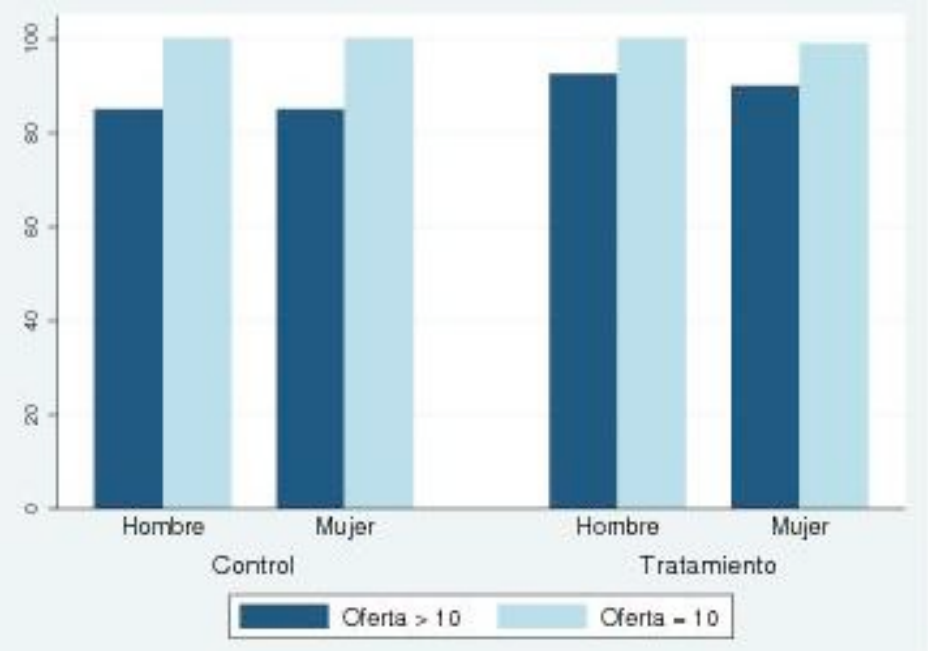

3.7. Creencias de los jugadores 2 en el $Y N G$

A la hora de preguntarle a los jugadores 2 del YNG sobre la probabilidad subjetiva que asignan a recibir una determinada oferta (PSR) vemos que estos, con independencia del tratamiento, atribuyen una probabilidad de recibir esa oferta superior al nivel objetivo de realización de dicho reparto (NOR).

\section{FIGURA 6 \\ MEDIANA DE LAS PSR Y NOR POR GÉNERO Y TRATAMIENTO}

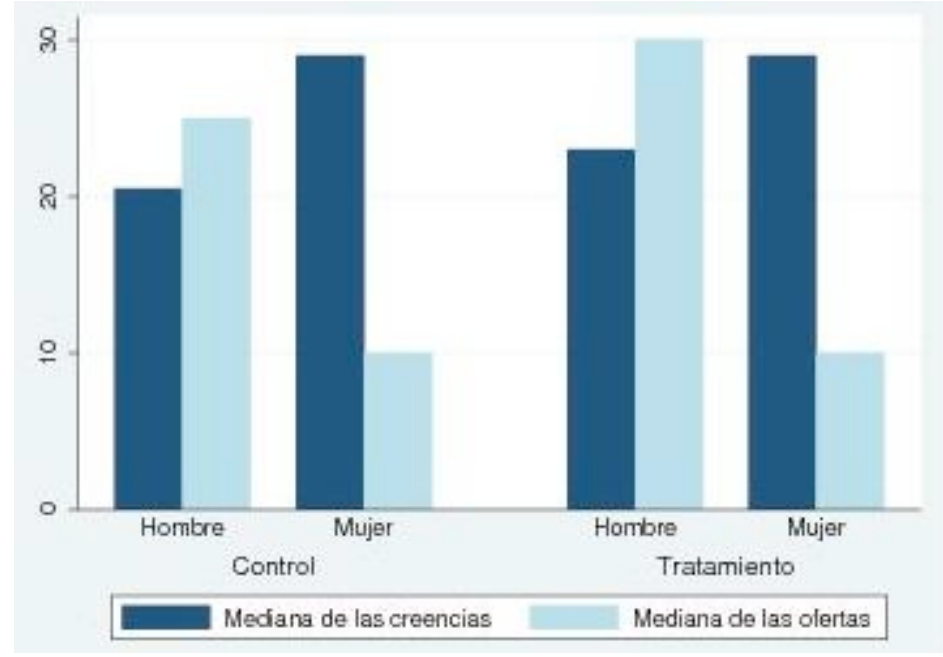


Sin embargo, cuando diferenciamos por género vemos que este resulta ser un mecanismo determinante, pues las mujeres asignan una probabilidad significativamente superior a la realización objetiva de ese reparto, mientras que los hombres asignan una PSR menor (test de Mann-Whitney; $z=-2,143 ; p=0,0321$ ). Y este último resultado se produce con independencia del tratamiento (ver figura 6).

\subsection{Síntesis de los principales resultados del YNG}

- Los jugadores 1 realizan repartos inferiores a los del UG y asignan una probabilidad muy alta a que su oferta sea aceptada: anticipan adecuadamente el comportamiento de los jugadores 2 .

- No existen diferencias significativas en las probabilidades subjetivas de aceptación de un determinado reparto que asignan los jugadores 1 atendiendo al sexo de los participantes y a la información de género.

- Los jugadores 2 del YNG, con independencia del tratamiento, atribuyen una probabilidad más alta de recibir una determinada oferta que no se corresponde con el nivel objetivo de realización de dicho reparto.

- Las jugadoras 2 del YNG asignan una probabilidad muy superior a la realización objetiva de ese reparto, mientras que los hombres asignan una PSR menor, con independencia del tratamiento.

\section{Conclusiones}

Las creencias de los sujetos son sensibles a la percepción del riesgo y se manifiestan de una forma coherente con la misma. Es decir, si los sujetos perciben riesgo dentro de un contexto económico ello se ve reflejado en las expectativas que los sujetos tienen sobre la interacción económica. Además, pequeñas variaciones como manejar información acerca del sexo del compañero, alteran las creencias de los individuos participantes. En el caso de este experimento, el efecto del tratamiento (información de género) sobre las expectativas subjetivas solo emerge en el juego del Ultimátum, cuando se asume el rol de proponer un determinado reparto, no igualitario, y se corre el riesgo de que este sea rechazado. Por tanto, podemos decir que en contextos estratégicos como el UG, anticipar el comportamiento de los compañeros (y por tanto toda información que arroje pistas sobre el mismo) se convierte en un elemento determinante a la hora de analizar una determinada situación.

Por otra parte, hombres y mujeres evalúan el riesgo de la interacción a la que se enfrentan de distinta manera. Las jugadoras 1 de este experimento en el contexto del UG tienen menos posibilidades de sufrir un rechazo que los jugadores 1, puesto que los repartos que han propuesto han sido mayores. Sin embargo, su mayor sensibilidad al riesgo hace que atribuyan las mismas probabilidades de éxito que sus compañeros masculinos. 
Cuando el riesgo no es un factor inherente a la interacción, como en el caso del jugador 2 en el UG o en el juego del Yes-or-No, las diferencias entre hombres y mujeres relacionadas con esta diferente sensibilidad al riesgo desaparecen, como cabe esperar. Sin embargo, cada sexo tiende a esperar de su compañero comportamientos distintos, las jugadoras 2 del YNG esperan recibir una determinada cantidad con una probabilidad mucho más alta de lo acontecido en la realidad, mientras que los jugadores 2 creen no recibir tal cuantía con una probabilidad inferior a la realidad. Quizás este hecho tenga que ver con el modo en que los individuos atribuyen comportamientos teniendo en cuenta sus propias preferencias sociales. Esta pregunta, sin embargo, escapa al objetivo central de este trabajo, que es el estudio de las creencias en contextos estratégicos en función del riesgo percibido.

\section{Referencias}

[1] BRAÑAS, P. y L. M. MILLER (2008). Instinctive response in the ultimatum game, Departmento de Teoría e Historia Económica, Universidad de Granada.

[2] BUCHAN, N. R., R. A. CROSON y S. SOLNICK. «Forthcoming. Trust and gender: An examination of behavior and beliefs in the Investment Game», Journal of Economic Behavior and Organization 62, pp. 466-476.

[3] COX, J. C. y C. A. DECK (2006). «When are women more generous than men?», Economic Inquiry 44, pp. 587-598.

[4] CROSON, R. y U. GNEEZY (2006, en prensa). «Gender differences in preferences», Journal of Economic Literature.

[5] ECKEL, C. C. y P. J. GROSSMAN (2001). «Chivalry and solidarity in Ultimatum Games», Economic Inquiry 39, pp. 171-188.

[6] ECKEL, C.C y P. J. GROSSMAN (1998). «Are women less selfish than men? Evidence from Dictator Experiments», Economic Journal 108, pp. 726-735.

[7] FISCHBACHER, U. (2007). «z-Tree: Zurich Toolbox for Ready-made economic experiments», Experimental Economics 10, pp. 171-178.

[8] GEHRIG, T., GÜTH, W., LEVNSK, R., OCKENFELS, A., USKE, T., y WEILAND, T. «Forthcoming. Buying a pig in a poke: An experimental study of unconditional veto power», Journal of Economic Psychology 28, pp. 692-703.

[9] GUTH, W., R. SCHMITTBERGER y B. SCHWARZE (1982). «An experimental analysis of Ultimatum Bargaining», Journal of Economic Behavior and Organization 3 , pp. 367-388.

[10] HOLM, H. (2000). «Gender-based focal points», Games and Economic Behavior 32, pp. 292-314.

[11] LEON-MEJIA, A. y LUIS M. MILLER (2007). The Devil is in the details. Sex differences in simple bargaining games, Jena Economic Research Papers in Economics 2007-069.

[12] SCHOTTER, A. y B. SOPHER (2007). «Advice and behavior in intergenerational ultimatum games: An experimental approach», Games and Economic Behavior 58, pp. 365-393.

[13] SELTEN, R. (1967). «Die Strategiemethode zur Erforschung des eingeschränkt rationalen Verhaltens im Rahmen eines Oligopolexperiments», Beiträge zur experimentellen Wirtschaftsforschung, I. Ed. por H. Sauermann, pp. 136-168, Tübingen: J.C.B. Mohr (Siebeck). 